\title{
On Top-k Recommendation using Social Networks
}

\author{
Xiwang Yang ${ }^{\dagger}$, Harald Steck ${ }^{\ddagger}$, Yang Guo ${ }^{\ddagger}$ and Yong $\mathrm{Liu}^{\dagger}$ \\ 'Polytechnic Institute of NYU, Brooklyn, NY, USA 11201 \\ ¥Bell Labs, Alcatel-Lucent, New Jersey \\ Email: xyang01@students.poly.edu, hsteck@gmail.com, Yang.Guo@alcatel-lucent.com, \\ yongliu@poly.edu
}

\begin{abstract}
Recommendation accuracy can be improved by incorporating trust relationships derived from social networks. Most recent work on social network based recommendation is focused on minimizing the root mean square error (RMSE). Social network based top-k recommendation, which recommends to a user a small number of items at a time, is not well studied. In this paper, we conduct a comprehensive study on improving the accuracy of top-k recommendation using social networks. We first show that the existing social-trust enhanced Matrix Factorization (MF) models can be tailored for top-k recommendation by including observed and missing ratings in their training objective functions. We also propose a Nearest Neighbor (NN) based top-k recommendation method that combines users' neighborhoods in the trust network with their neighborhoods in the latent feature space. Experimental results on two publicly available datasets show that social networks can significantly improve the top-k hit ratio, especially for cold start users. Surprisingly, we also found that the technical approach for combining feedback data (e.g. ratings) with social network information that works best for minimizing RMSE works poorly for maximizing the hit ratio, and vice versa.
\end{abstract}

\section{Categories and Subject Descriptors}

H.3.3 [Information Search and Retrieval]: Information Filtering

\section{General Terms}

Algorithms, Design, Measurement, Experimentation

\section{Keywords}

Recommender System, Social Network, Matrix Factorization

${ }^{*}$ Now at Netflix Inc. Work was done while at Bell Labs.

Permission to make digital or hard copies of all or part of this work for personal or classroom use is granted without fee provided that copies are not made or distributed for profit or commercial advantage and that copies bear this notice and the full citation on the first page. To copy otherwise, to republish, to post on servers or to redistribute to lists, requires prior specific permission and/or a fee.

Copyright 20XX ACM X-XXXXX-XX-X/XX/XX ...\$10.00.

\section{INTRODUCTION}

The idea of recommender systems (RS) is to automatically suggest items to each user that $\mathrm{s} /$ he may find appealing, e.g. see [1] for an overview. Traditional collaborative filtering approaches predict users' interests by mining user rating history data $[3,9,16-20]$. In real life, people often resort to their friends for recommendations in real life. It is therefore tempting to improve RS by incorporating information on the trust relationships between users in social networks. In the literature, e.g. [4, 6-8, 11, 12, 15], it was shown that using social network information in addition to feedback data (e.g. ratings) can significantly improve recommendation accuracy. While there is some improvement on the average recommendation accuracy over all users, the improvement is particularly significant for the so-called cold users, who have provided only very little feedback (e.g. very few ratings) $[5,7,8,15]$. Most of the existing social network based recommender systems are optimized for the root mean square error (RMSE), which has enjoyed perhaps the largest popularity among the various accuracy measures in the recommender literature. On the other hand, top-k recommendation, a small number $k$ of items been recommended to a user at a time is pervasive in Real-World recommendation task.

In this paper, we provide a comprehensive study on improving the accuracy of top-k RS using social networks. Towards this goal, we first show that the existing social-trustenhanced Matrix Factorization (MF) models [7,8,11,12] can be conveniently tailored for top-k recommendation by extending their training objective functions to include both the observed ratings and the missing ratings. For the Nearest Neighbor (NN) based top-k recommendation, we propose to combine users' neighborhood in their trust network with their neighborhood in the user latent feature space derived from matrix factorization considering MNAR. To generate top-k recommendation from a combined neighborhood, instead of taking the weighted average over only the observed ratings, we propose to use voting-based algorithm as a simple approach to consider both observed and missing ratings.

To assess the performance of the proposed social-networkbased top-k RSes, we then undertake a comprehensive comparison study using two publicly available real-world data sets: Epinions and Flixster. The major findings are:

1. Trust information significantly improves top- $k$ hit ratio when incorporated properly both in MF and NN models.

2. Our proposed social network based NN models and RS 
trained with our modified objective function considerably outperform the only published top- $k$ approach using social network information in recent literature [7], an NN approach.

3. Among the various ways of combing feedback data with social network information, the one that was found to be worst with respect to RMSE turns out to be the best concerning the top- $k$ hit ratio. This illustrates that the technical details of minimizing RMSE can be very different from the ones that work to optimize top$k$ hit ratio.

This paper is organized as follows. Some related work is discussed in section 2. Section 3 outlines the top- $k$ models using social network information. In particular, in Sections 3.1.1 to 3.1.3, we outline various matrix factorization (MF) models for combining feedback data and social trust information that were proposed in the literature. As they are typically geared towards RMSE, we modify their training objectives functions towards the top- $k$ hit ratio. We propose a set of social network based NN models in Section 3.2. In Section 4, we present the comprehensive comparison study of the various approaches-with respect to the top- $k$ hit ratio. The paper is concluded in Section 5 .

\section{RELATED WORK}

\subsection{Recommendation Task}

We consider the following Real-World Recommendation Task: for each user, the recommender system has to recommend a small number, say $k$, of items from among all available items. One may distinguish between two slightly different variants of this task: (a) all items are eligible for recommendation, including the items that have been rated ${ }^{1}$ by the user in the past (this assumes that a user may consume an item possibly several times, e.g. listen to a song on the online radio); or (b) only those items, which have not been rated by the user in the past, are eligible for recommendation (this assumes that a user consumes an item at most once, e.g. purchase of a movie DVD).

A meaningful offline test ideally should provide a good approximation to the utility function optimized by the deployed system (e.g. user satisfaction, increase in sales). This immediately suggests the corresponding procedures for offline testing on available data:

- all items: for each user, all items are considered, whether rated or not by the user (in the training or test set).

- all unrated items: for each user, only those items are considered that have not been rated by the user in the training set. Note that this contains items with and without ratings in the test data.

Due to the data sparsity, the difference between these two variants is expected to be small, as confirmed by our experiments. We hence will only report results concerning the second variant in this paper.

The user's selection bias causes the observed feedback (e.g. ratings, purchases, clicks) in the data to be missing not at

${ }^{1}$ For simplicity, we use 'ratings' as a synonym for feedback in this paper. As will become clear, the presented approach is applicable to both explicit and implicit feedback data. random (MNAR). This is an important issue in practice, but largely ignored in the literature (the few exceptions include $[2,13,14,21])$. Selection bias may result from user's tendency to rate only the items they like or know. Compared to the (unknown) distribution over (a random subset of) all ratings, the distribution of observed ratings is skewed due to the selection bias. As the $k$ recommended items have to be chosen from all items (that were not rated in the training set), this unknown distribution influences the recommendation accuracy, and hence user satisfaction in practice. The top- $k$ hit ratio provides a direct assessment of a recommender system's accuracy [21]. In contrast, RMSE on the observed data is agnostic to the selection bias, as the data in the training set and the test set are from the same skewed distribution.

Recent work by Marlin et al. [13,14] provided empirical evidence that the data typically used for training and testing recommender systems indeed exhibit a significant selection bias, i.e., the ratings are missing not at random: their histograms of the distribution of ratings in the Yahoo!LaunchCast data show that low ratings are much more likely to be missing from the observed data than high ratings (see Figure 2 in [13]).

If the ratings in the available data had been missing at random (MAR), unbiased results could have been expected from the common test procedures using observed ratings only. It is shown in [21] that the top- $k$ hit ratio or recall has desirable properties when applied to all (unrated) items in MNAR test data. Note that approaches that perform well with respect to RMSE on the observed ratings may perform poorly with respect to the top- $k$ hit-ratio on all items $[2,10,21]$.

\subsection{Top- $k$ Hit-Ratio}

As to compute the top- $k$ hit ratio or recall, for each user $u$, we rank the items $i$ according to the predicted rating $\hat{R}_{i, u}$. Since the predicted rating is continuous, the ranking list is unique. Otherwise, ties are broken at random. An item is defined as relevant to a user in the test set if $\mathrm{s} / \mathrm{he}$ finds it appealing or interesting (e.g., the assigned rating in the test data is above a certain threshold). For instance, in our experiments with Epinions data, the rating values range from $1, \ldots, 5$ stars, and we consider 5 -star ratings as relevant (i.e. the user definitely liked these items), while other rating values and missing rating values are considered not relevant. Other choices led to similar results. Now the top- $k$ hit ratio or recall can be defined as the fraction of relevant items in the test set that are in the top- $k$ of the ranking list, denoted by $N(k, u)$, from among all relevant items, $N(u)$. For each user $u$, the top-k hit ratio is given by

$$
\mathrm{H}(k, u)=\frac{N(k, u)}{N(u)},
$$

which can be aggregated over all users to obtain the average top- $k$ hit ratio or recall for the test set. The recall is computed as follows:

$$
\text { recall }=\frac{\sum_{u} N(k, u)}{\sum_{u} N(u)},
$$

Note that a higher top-k hit ratio or recall is better. In our experiments, the evaluation metric is recall.

\subsection{Top- $k$ Matrix Factorization}


AllRank [21] was found to be the best approach to optimizing the top- $k$ hit ratio on various data sets in the literature $[2,10,21]$, outperforming various neighborhood models and the SVD++ [10], among others. It is based on a lowrank matrix-factorization (MF) model: the matrix of predicted ratings $\hat{R} \in \mathbb{R}^{u_{0} \times i_{0}}$, where $i_{0}$ denotes the number of items, and $u_{0}$ the number of users, is modeled as

$$
\hat{R}=r_{m}+Q P^{\top},
$$

with matrices $P \in \mathbb{R}^{i_{0} \times j_{0}}$ and $Q \in \mathbb{R}^{u_{0} \times j_{0}}$, where $j_{0} \ll$ $i_{0}, u_{0}$ is the rank; and $r_{m} \in \mathbb{R}$ is a (global) offset. Ideally, one would directly optimize the top- $k$ hit ratio in the training step. Unfortunately, this is computationally prohibitive. We hence resort to optimizing the square error:

$$
\sum_{\text {all } u \text { all } i} W_{u, i} \cdot\left(R_{u, i}^{\mathrm{o} \& \mathrm{i}}-\hat{R}_{u, i}\right)^{2}+\lambda\left(\|P\|_{F}^{2}+\|Q\|_{F}^{2}\right)
$$

The key here is to train the model on all items by summing not only over the observed ratings. $\lambda>0$ is a regularization parameter. We use the Frobenius norm, denoted by $\|\cdot\|_{F}$, to regularize the learned matrices $P$ and $Q$. Note that this is slightly different from the original AllRank version [21], which uses the trace norm regularization. The difference between these two kinds of regularization are discussed in detail concerning RMSE in [19]. Concerning the top- $k$ hit ratio, we found the resulting difference to be rather small. The use of the Frobenius norm here is motivated merely by the fact that we want to be consistent with the recommender systems that use social network information in the literature $[8,11,12]$, which use the Frobenius norm regularization. The ratings predicted by the model are denoted by $\hat{R}_{u, i}$ (see (3)); and $R_{u, i}^{\text {o\&i }}$ equals the actual rating value in the training data if observed for user $u$ and item $i$; otherwise the value $R_{u, i}^{\mathrm{o} \& \mathrm{i}}=r_{m}$ is imputed. The key is in the training weights,

$$
W_{u, i}= \begin{cases}1 & \text { if } R_{u, i}^{\text {obs }} \text { observed } \\ w_{m} & \text { otherwise }\end{cases}
$$

It is crucial that the weight assigned to the imputed ratings is positive, ie $w_{m}>0$ in AllRank [21]. In contrast, the usual optimization of the RMSE test-measure is obtained by training with $w_{m}=0$. This seemingly small difference has the important effect that AllRank is trained on all items, while RMSE-approaches are trained only on the observed ratings. AllRank achieves a considerably larger top- $k$ hitratio or recall on all items than the various state-of-the-art approaches (see results in [21] and compare to $[2,10]$ ).

Alternating least squares ( $A L S$ ) can be used for computationally efficient training of AllRank [21]. Alternatively, stochastic gradient descent with sub-sampling of the imputed ratings may be used, like in [16], which typically leads to (slightly) degraded hit ratios, but is computationally more efficient than ALS for certain models.

In $A L S$, for fixed $Q$, the matrix $P$ that minimizes (4) can be calculated using the usual necessary condition (equate gradient to zero), and solving for $P_{i}$, for each item $i$. This results in the following update equation for each row $i$ of $P$ :

$$
P_{i, .}=\left(R_{\cdot, i}^{\mathrm{o} \& \mathrm{i}^{\top}}-r_{m}\right) \tilde{W}^{(i)} Q\left(Q^{\top} \tilde{W}^{(i)} Q+\lambda I\right)^{-1},
$$

where the dot in the index of a matrix refers to the vector of all entries; $\tilde{W}^{(i)}=\operatorname{diag}\left(W_{i, .}\right) \in \mathbb{R}^{u_{0} \times u_{0}}$ is the diagonal matrix containing the $i^{\text {th }}$ row of weight matrix $W ; I \in \mathbb{R}^{j_{0} \times j_{0}}$ is the identity matrix. While the diagonal matrix $\tilde{W}^{(i)}$ may appear to be of computationally prohibitive size, (6) can be computed efficiently [21]. The update equation for each row $u$ of $Q$ is analogous.

\section{TOP- $K$ RECOMMENDER SYSTEMS US- ING SOCIAL NETWORKS}

Recommender systems using social network information were mainly developed to optimize RMSE on observed ratings, e.g. $[6,8,11,12,15]$. Various approaches are used. While neighborhood $[4,7]$ and random walk [6] methods were used on the social network graph, matrix factorization methods were found to be the most accurate model also in the context of social network information $[8,11,12]$.

For this reason, we start with matrix factorization (MF) approaches using social network information $[8,11,12]$, and modify them as to optimize the top- $k$ hit ratio (rather than the original RMSE). Each of the three models and its modification is outlined in the following sub-sections. Other than $\mathrm{MF}$ approaches, we also consider nearest neighbor (NN) approaches. In fact, in recent recommender literature, the only top- $k$ approach using social network information is a NN method [7] to the best of our knowledge. In Section 3.2, we develop several variants of $\mathrm{NN}$ approaches by adopting user latent features derived from MF optimized for top-k hit ratios.

\subsection{Top- $k$ MF using Social Networks}

In the following subsection, we briefly review the existing MF approaches in the literature that combine rating data with social network information $[8,11,12]$. As to optimize the top- $k$ hit ratio (rather than RMSE), we modify their training function as to account for all items, rather than the observed ratings only, analogous to AllRank in Section 2.3 .

The social network information is represented by a matrix $S \in \mathbb{R}^{u_{0} \times u_{0}}$, where $u_{0}$ is the number of users. The directed and weighted social relationship of user $u$ with user $v$ (e.g. user $u$ trusts/knows/follows user $v$ ) is represented by a positive value $S_{u, v} \in(0,1]$. An absent or unobserved social relationship is reflected by $S_{u, v}=s_{m}$, where typically $s_{m}=0$.

\subsubsection{Social Recommendation (SoRec) Model}

Social Recommendation (SoRec) was introduced in [12]. In this model, the social network matrix $S$ (see beginning of Section 3.1 ) may be slightly modified as follows [12]:

$$
S_{u, v}^{*}=S_{u, v} \sqrt{\frac{d_{v}^{-}}{d_{u}^{+}+d_{v}^{-}}},
$$

where $d_{u}^{+}$is the out-degree of user $u$ in the social network (i.e. the number of users whom $u$ follows/trusts), and $d_{v}^{-}$ is the in-degree of user $v$ in the network (ie the number of users who follow/trust user $v$ ).

The predicted ratings are obtained from the model as follows:

$$
\hat{R}=r_{m}+Q P^{\top},
$$

where $P$ and $Q$ are the low-rank matrices of the model. While this is the same equation as (3), which does not use social network information, note that the information from 
the social network is implicitly present in the learned matrices $P$ and $Q$. Besides the rating data, also the social network information is used for training this model. The social relationships are predicted as follows:

$$
\hat{S}^{*}=s_{m}+Q Z^{\top},
$$

where $Z \in \mathbb{R}^{u_{0} \times j_{0}}$ is a third matrix in this model, besides $P$ and $Q$. Note that the matrix $Q$ is shared among the two equations (7) and (8). Due to this constraint, one can expect $Q$ (i.e. the user profiles $Q_{u}$ for each user $u$ ) to reflect information from both the ratings and the social network as to achieve accurate predictions for both. Note that the matrix $Z$ is not needed for predicting rating values, and hence may be discarded after the matrices $P$ and $Q$ have been learned. Both (7) and (8) are combined as follows in the training objective function. Analogous to Section 2.3, we modify the training function as to optimize the top- $k$ hit ratio (instead of RMSE):

$$
\begin{aligned}
& \sum_{\text {all } u \text { all } i} \sum_{u, i} \cdot\left(R_{u, i}^{\mathrm{o \& i}}-\hat{R}_{u, i}\right)^{2} \\
+ & \sum_{\text {all } u \text { all } v} \sum_{u, v} W_{u, v}^{(S)} \cdot\left(S_{u, v}^{*}-\hat{S}_{u, v}^{*}\right)^{2} \\
+ & \lambda\left(\|P\|_{F}^{2}+\|Q\|_{F}^{2}+\|Z\|_{F}^{2}\right),
\end{aligned}
$$

where $\|\cdot\|_{F}$ denotes the Frobenius norm of the matrices, and $\lambda$ is the usual regularization parameter. $W_{u, i}$ and $R_{u, i}^{\mathrm{o} \& \mathrm{i}}$ are defined as in the AllRank model, see Section 2.3. The term concerning the social network (in the second line) is analogous to the first term concerning the ratings. In particular, the absent or unobserved social links are treated analogous to the missing ratings in AllRank, i.e. we impute the value $s_{m}$ with weight $w_{m}^{(S)}$. Like $W_{u, i}$ in $(5), W_{u, v}^{(S)}$ is defined as follows:

$$
W_{u, v}^{(S)}=\gamma \cdot\left\{\begin{array}{ll}
1 & \text { if } S_{u, v}^{*} \text { observed } \\
w_{m}^{(S)} & \text { otherwise }
\end{array},\right.
$$

where $\gamma \geq 0$ determines the weight of the social network information compared to the rating data. Obviously, $\gamma=0$ corresponds to the extreme case where the social network is ignored when learning the matrices $P$ and $Q$. As $\gamma$ increases, the influence of the social network increases. The effect is that the user profiles $Q_{u}$ and $Q_{v}$ of two users $u$ and $v$ become more similar to each other if they are friends. While only positive social relationships are considered in the original model [12], we note that this model allows also for negative values of $S_{u, v}$, representing e.g. distrust among users.

Note that the rating matrix $R^{\text {o\&i }}$ and the social network matrix $S^{*}$ may be combined into a single matrix (and the weight matrices analogously): $\left[R^{\text {o\&i }} S^{*}\right]$ and $\left[W W^{(S)}\right]$. When these two matrices are used in AllRank, SoRec training is formally identical to AllRank training. The social relationships hence take the place of virtual rating values, where friends appear to have rated the same virtual items (i.e. common friends). Apart from that, when the training objective is re-written in this form, it is clear that the same ALS update equations can be applied as for AllRank (see Section 2.3).

\subsubsection{Social Trust Ensemble (STE) Model}

Recommendation with Social Trust Ensemble (STE) was introduced in [11]. The predicted ratings are obtained from the model, comprising the matrices $P \in \mathbb{R}^{i_{0} \times j_{0}}$ and $Q^{u_{0} \times j_{0}}$, as follows:

$$
\hat{R}_{u, i}=r_{m}+\alpha Q_{u} P_{i}^{\top}+(1-\alpha) \sum_{v} S_{u, v} Q_{v} P_{i}^{\top},
$$

where we omitted the logistic function, as we found its effect rather negligible in our experiments. The reason is that only the ranking/order of the predicted rating values is important for the top- $k$ hit ratio, while it is irrelevant if the predicted rating values are confined to valid rating values (e.g. the interval $[1,5]$ stars). The trade-off between the feedback data (ratings) and the social network information is determined by $\alpha \in[0,1]$. Obviously, the social network information is ignored for $\alpha=1$, while $\alpha=0$ assigns the highest possible weight to the social network information. Intermediate values of $\alpha$ result in a weighted combination of the information from both sources. (11) is equivalent to the matrix notation

$$
\hat{R}=r_{m}+S_{\alpha} Q P^{\top},
$$

where $S_{\alpha}=\alpha I+(1-\alpha) S$, and $I$ is the identity matrix. Analogous to Section 2.3, the modified training function geared towards the top- $k$ hit ratio then reads as follows:

$$
\sum_{\text {all } u \text { all } i} \sum_{u, i} \cdot\left(R_{u, i}^{\mathrm{o} \& \mathrm{i}}-\hat{R}_{u, i}\right)^{2}+\lambda\left(\|P\|_{F}^{2}+\|Q\|_{F}^{2}\right),
$$

where $\|\cdot\|_{F}$ denotes the Frobenius norm. $W_{u, i}$ and $R_{u, i}^{\mathrm{o} \& \mathrm{i}}$ are defined as in the AllRank model, see Section 2.3. We found this objective function to be computationally inefficient to optimize using ALS. The update equation for $Q_{u}$, reads:

$$
\begin{aligned}
Q_{u, .}= & \left(\varepsilon Q_{u}^{o l d}+\alpha\left(R_{u, .}^{o \& i}-r_{m}\right) \tilde{W}^{(u)} P\right. \\
& \left.-(1-\alpha) \sum_{v \mid u \in N_{v} \text { all } i} \sum_{v, i}\left(R_{v, i}^{o \& i}-\hat{R}_{v, i}\right) S_{v, u} P_{i, .}\right) \\
& \cdot\left(\alpha P^{\top} \tilde{W}^{(u)} P+(\lambda+\varepsilon) I\right)^{-1}
\end{aligned}
$$

where $N_{u}$ is the set of users that $u$ trusts. Note that we added an additional term on either side, $\varepsilon Q_{u, \text {. and }} \varepsilon Q_{u, .}^{\text {old }}$, respectively. We found that, for the STE model, ALS gets easily stuck in a local optimum without this term. Using an appropriate value $\varepsilon>0$, effectively results in a smaller step size of ALS, requiring more iterations until convergence, but helping avoid local optima. It is very expensive to compute the second term in the equation. As an approximation, we just sum up the observed ratings for all users $v \mid u \in N_{v}$, instead of summing up all items. As the update equation of $P$, it is identical to $(6)$.

\subsubsection{Social MF Model}

The SocialMF model was proposed in [8], and was found to outperform SoRec and STE with respect to RMSE. Each of the rows of the social network matrix $S$ has to be normalized to 1 , resulting in the new matrix $S^{*}$ with $S_{u, v}^{*} \propto S_{u, v}$, and $\sum_{v} S_{u, v}^{*}=1$ for each user $u$.

The predicted ratings are obtained from the model, comprising the matrices $P \in \mathbb{R}^{i_{0} \times j_{0}}$ and $Q^{u_{0} \times j_{0}}$, as follows:

$$
\hat{R}=r_{m}+Q P^{\top},
$$

where we again omitted the logistic function, as we found its effect rather negligible in our experiments. Analogous to 
Section 2.3, we modified the training function in [8] as to optimize the top- $k$ hit ratio (instead of RMSE):

$$
\begin{aligned}
& \sum_{\text {all } u \text { all } i} \sum_{u, i} \cdot\left(R_{u, i}^{\mathrm{o \& i}}-\hat{R}_{u, i}\right)^{2} \\
+ & \beta \sum_{\text {all } u}\left(\left(Q_{u}-\sum_{v} S_{u, v}^{*} Q_{v}\right)^{\top}\left(Q_{u}-\sum_{v} S_{u, v}^{*} Q_{v}\right)\right) \\
+ & \lambda\left(\|P\|_{F}^{2}+\|Q\|_{F}^{2}\right)
\end{aligned}
$$

The tradeoff between the feedback data (ratings) and the social network information is determined by $\beta \geq 0$. Obviously, the social network information is ignored for $\beta=0$, while increasing values of $\beta$ shift the tradeoff more and more towards the social network information. The term in the second line constitutes a constraint that a user profile $Q_{u}$ should be similar to the (weighted) average of his/her friends' profiles $Q_{v}$ (measured in terms of the square error).

(16) can be optimized by stochastic gradient descent using the update equations (13) and (14) in [8]; however, it has to be computed not only for the available ratings, but also for the imputed values. Due to the sheer number of imputed values, this may become computationally inefficient. As an approximation, one may use a sub-sample of the imputed values for efficient computations (cf also [16]). Alternatively, (16) can be optimized by ALS, like Allrank (see Section 2.3). For fixed $P$, the update equation for row $u$ of matrix $Q$ is determined by the constraint that the derivative of (16) equals zero, which results in

$$
\begin{aligned}
0= & Q_{u, .}\left(P^{\top} \tilde{W}^{(u)} P+\lambda I\right) \\
& -\left(R_{u, .}^{\mathrm{o \& i}}-r_{m}\right) \tilde{W}^{(u)} P \\
& -\beta\left(I-S^{* \top}\right)_{u, .}\left(I-S^{*}\right) Q
\end{aligned}
$$

where $\tilde{W}^{(u)}=\operatorname{diag}\left(W_{., u}\right) \in \mathbb{R}^{i_{0} \times i_{0}}$ is the diagonal matrix containing the $u^{\text {th }}$ column of the weight matrix $W$. Due to the social network matrix $S^{*}$, this equation cannot be solved analytically for $Q$. Given that ALS is an iterative procedure, one may replace $Q$ in the third line by its value from the previous iteration, $Q^{\text {old }}$. Then this equation can be solved for $Q_{u, .}$, resulting in the update equation

$$
\begin{aligned}
Q_{u, .}= & \left(\left(R_{u, .}^{\mathrm{o} \& \mathrm{i}}-r_{m}\right) \tilde{W}^{(u)} P\right. \\
& \left.+\beta\left(I-S^{* \top}\right)_{u, .}\left(I-S^{*}\right) Q^{\mathrm{old}}+\varepsilon Q_{u, .}^{\mathrm{old}}\right) \\
& \cdot\left(P^{\top} \tilde{W}^{(u)} P+(\lambda+\varepsilon) I\right)^{-1}
\end{aligned}
$$

where we again added the same additional terms, $\varepsilon Q_{u}$.. and $\varepsilon Q_{u, .}^{\text {old }}$, as to avoid local optima. As the update equation of $P$ does not involve the social network matrix $S^{*}$, it is identical to $(6)$.

\subsection{Nearest Neighbor Methods}

In a NN method, top-k recommendations are generated not from all items, but only from items "liked" by a subset of users who are "nearest" (under certain distance metric) to the target user. The neighborhood of a user can be calculated using collaborative filtering, or it can be just a set of directly or indirectly connected friends in a social network. This makes it convenient to incorporate social trust into NN based top-k recommendation.
Basically, NN based RS is quite a different approach from MF based RS in terms of real system deployment. In RealWorld systems, there are lots of user's feedbacks every day, e.g., as it is reported that there are billions of the like buttons served daily in facebook. NN based RS enjoys a unique advantage in that it can incrementally integrate user's new feedback into recommendation. Nearest neighbors of a user is fixed between two sequential neighborhood update, so one user's new feedbacks is able to influence the recommendation to its neighbors in real time. While, in MF based approach, in order to integrate user's new feedbacks, it requires new matrix factorization which is not efficient when deployed in real systems. Due to this difference, we think its meaningful and necessary to study NN models.

To the best of our knowledge, [7] is the only work that incorporates social network into NN based top- $k$ recommender system. Two neighborhood based approaches are studied in [7] and their performance are comparable. We thus select one model there, termed as Trust-cf, as the baseline in our comparison.

In Trust-cf, Breadth First Search (BFS), starting from a source user $u$, is performed to obtain a set of trusted neighbors, namely trusted neighborhood. Meanwhile, collaborative filtering $(\mathrm{CF})$ neighborhood consists of users who are close to the source user $u$ in terms of Pearson Correlation coefficient. The items rated highly by users in either neighborhoods are considered to be candidates for top- $k$ recommendation. Trust-cf calculates the predicted rating for a candidate item as the weighted average of all observed ratings in the two neighborhoods. The weight for a user in the trusted neighborhood is set to $1 / d_{v}$, where $d_{v}$ is the depth of user $v$ from the source user $u$ in the trust network. The weight for a user in the CF neighborhood is the Pearson Correlation coefficient between this user and the source user. If an item has predicted ratings from both neighborhoods, two predicted ratings are combined using weighted average with weights proportional to the neighborhood size for this item. Finally, trust-cf sorts all the candidate items by their predicted ratings and recommends the top- $k$ to the source user.

We propose a set of social network based NN approaches to achieve high top- $k$ hit ratio by considering both social trust and MNAR. We always denote by $k_{1}$ the number of nearest users identified by the Collaborative Filtering (CF) approach, and by $k_{2}$ the number of trusted users identified by the social network based approach.

- CF-ULF approach. CF-ULF uses AllRank to obtain the user latent features. The users are then clustered in the user latent feature space using the Pearson correlation coefficient. The $k_{1}$ users nearest to the source user $u$ are identified. The relevant items of these nearest users are voted to form the top- $k$ recommended items. The voting for the candidate items are computed as follows:

$$
\text { Vote }_{u, i}=\sum_{v \in N_{u}} \sum_{i} \operatorname{sim}(u, v) \delta_{i \in I_{v}}
$$

where $\delta$ is the Kronecker delta; $I_{v}$ denotes the set of relevant items of user $v$; and $N_{u}$ is the set of $k_{1}$ nearest neighbors of user $u$ (as determined by the Pearson correlation). Vote $_{u, i}$ is the voting concerning item $i$ for user $u$; the $k_{1}$ nearest neighbors of user $u$ are weighted according to their similarity $\operatorname{sim}(u, v)$ with user $u$, measured in terms of the Pearson correlation coefficient between user $u$ and $v$ (in user latent 
feature space).

- PureTrust approach. Pure Trust approach employs the breadth-first search (BFS) in the social network to find $k_{2}$ trusted users to the source user $u$.

The voting scheme is similar to the scheme employed in $C F-U L F$.

$$
\text { Vote }_{u, i}=\sum_{v \in N_{u}^{(t)}} \sum_{i} w_{t}(u, v) \delta_{i \in I_{v}},
$$

where $N_{u}^{(t)}$ is the set of trusted users of $u$, and by $w_{t}(u, v)$ the voting weight from user $v$. The value of $w_{t}(u, v)$ is set to be $1 / d_{v}$, where $d_{v}$ is the depth of user $v$ in the BFS tree rooted at user $u$.

- Trust-CF-ULF approach. Trust-CF-ULF approach is the combination of user latent feature space based collaborative filtering (CF-ULF) approach and social network based approach. The value of $k_{1}$ is set to be equal to the value of $k_{2}$ in Trust-CF-ULF. In this approach, we firstly find $k_{1}$ closest neighbors from the $\mathrm{CF}$ neighborhood, then find $k_{2}$ closet neighbors from the trust neighborhood which are not in the $k_{1}$ set. Then users in the combined neighborhood vote for their relevant items. $w(u, v)$ is defined as following:

$$
\text { Vote }_{u, i}=\sum_{v \in N_{u}^{(c)}} \sum_{i} w(u, v) \delta_{i \in I_{v}},
$$

where, $N_{u}^{(c)}$ is the combined neighborhood.

$$
w(u, v)=\left\{\begin{array}{rl}
\operatorname{sim}(u, v) & \text { if } v \in N_{u} \\
w_{t}(u, v) & \text { if } v \in N_{u}^{(t)}
\end{array},\right.
$$

- Trust-CF-ULF-best approach. Trust-CF-ULF-best improves upon Trust-CF-ULF by dynamically tuning the value of $k_{1}$ and $k_{2}$ so as to obtain the best recall results.

The main differences between our proposed NN methods and Trust-cf are: 1) Our CF neighbors are derived from user latent features obtained from AllRank, which is expected to have higher top-k hit ratio than the Pearson correlation coefficient based only on observed ratings; 2) We use voting, instead of the weighted averaging of the observed ratings, to construct top-k recommendations. Voting can be treated as the simplest approach to consider all items with and without ratings. As will be shown in Section 4.2.4, our NN models perform much better than the existing social network based NN models.

\section{EXPERIMENTS}

In this section, we perform experiments for the proposed top-k RSes on Epinions ${ }^{2}$ and Flixster ${ }^{3}$ datasets. We focus on the top- $k$ hit ratio or recall as a more realistic measure for testing recommendation accuracy (as motivated in Section 2). Concerning the three MF models, SoRec, STE and SocialMF (see Section 3.1), we used rank $j_{0}=10$, like in $[8,11,12]$. We find that Trust information significantly improves top- $k$ hit ratio when incorporated properly both in MF and NN models. We also find that our proposed NN based RS and MF models trained with our modified objective function considerably outperform the existing top- $k$ approach using social network information in recent literature [7]. Moreover, among the three models for combining

\footnotetext{
${ }^{2}$ http://www.epinions.com/

${ }^{3}$ http://www.flixster.com/
}

rating data with social network information, the model with the worst performance concerning RMSE surprisingly turns out to achieve the best top- $k$ hit ratio. This illustrates that approaches that work well for the vastly popular RMSE are not necessarily useful for optimizing the more realistic top- $k$ hit ratio or recall.

\subsection{Dealing with High Computation Cost}

Training on all items admittedly increases the computation complexity, which is another key performance metric, other than accuracy, in designing recommender systems. Good recommendation algorithms not only need to provide accurate results, but also need to be scalable to large problems. To work with two large real-world datasets, we conducted our experiments on a Linux server with four E5640 Intel Xeon CPUs. Each CPU has four cores, and each core has 12.3 MB cache. The shared memory size is $12 \mathrm{~GB}$. We implemented multi-thread $\mathrm{C}++$ programs to parallelize large-scale matrix operations encountered in model training and parameter optimization. The running times for different models ranges from seconds to hours. For the STE model, we could not afford the computation cost to get the exact optimal solution, and we resorted to approximation methods. We found that the stochastic gradient descend and gradient descend methods easily got stuck in local minima when training with missing ratings, while ALS performed better in many cases.

\subsection{Experiments on Epinions Dataset}

\subsubsection{Dataset}

Epinions is a consumer opinion site where users review various items, such as cars, movies, books, software, etc., and assign ratings to the items. The ratings are in the range of $1(\min )$ to $5(\max )$. Users also assign trust values (i.e. a value of 1) to other users whose reviews and/or ratings they find valuable. No trust value indicates that a user either does not know the other, or distrusts him. We used the Epinions dataset ${ }^{4}$ published by the authors of [22] in our experiments.

The Epinions data set consists of 71,002 users with a total number of 104,356 rated items. The total number of reviews is 571,235 , and the total number of pairwise, directed trust relationships is 508,960 . In our experiments, the data set is divided into two sub-sets: the training set and the test set. For users with less than five ratings, one randomly selected rating is put into the test set. For users with five ratings or more, $10 \%$ of the randomly selected ratings are moved to the test set. We define cold user as user who had rated fewer than 5 items. We further split the test set randomly into two disjoint sets of equal size. The first test set is used for cross-validation during training as to determine the tuning parameters in our objective function. The second test set is used as a truly held-out data set for final evaluation of the trained model. We report the result of testing the second test set. We consider 5-star ratings as relevant ${ }^{5}$ to a user, i.e. the user definitely likes these items, and report the recall test results for the top 500 items (as defined in Section 2). The reason we set $k=500$ is as

\footnotetext{
${ }^{4}$ http://alchemy.cs.washington.edu/data/epinions/

${ }^{5}$ Considering both 4 and 5 star ratings as relevant, experiments showed similar differences among the various approaches.
} 


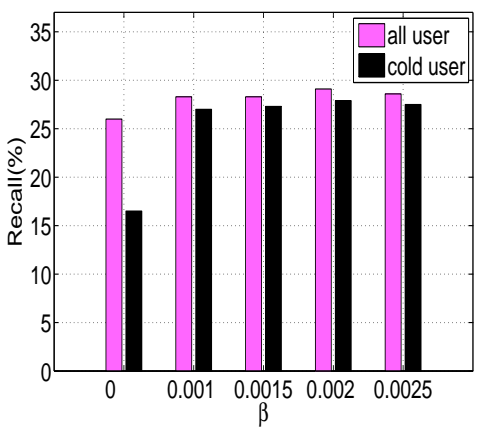

(a) SocialMF model

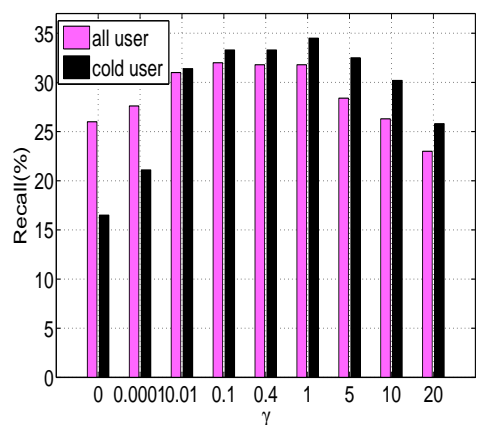

(b) SoRec model

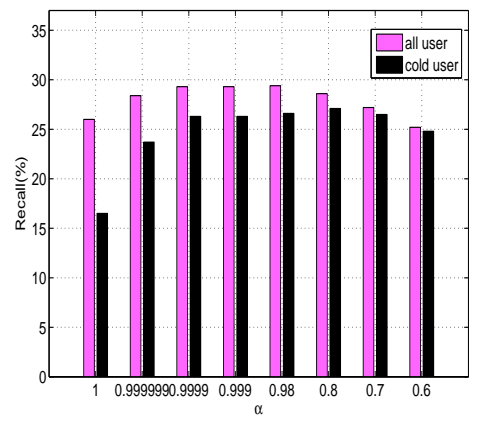

(c) STE model

Figure 1: Top-500 Recall for social-network-based matrix-factorization models on Epinions data.

follows. In Epinions dataset, there are much more number of items than users which is different from many other data sets, e.g. Netflix dataset ${ }^{6}$. Thus, using a small value for $k$ will produce generally poor results for all compare methods. Actually, we have performed experiments for $k=5$. The recall of modified SoRec model on all user and cold user were $2.06 \%$ and $2.45 \%$ respectively, and the recall of modified No Trust on all user and cold user were $1.31 \%$ and $0.93 \%$ respectively. Nonetheless, we show the results of recall as the value of $k$ changes in Figure 6 .

\subsubsection{Recall for MF Models}

We found the following tuning parameters of the training objective functions for the MF models (see Section 2.3) to result in the highest recall: $\lambda=0.4, r_{m}=-1, w_{m}=0.0002$ for all models; the optimal tradeoff between the rating data and the social network information is determined by the parameters $\beta$ for SocialMF, $\alpha$ for STE, and $\gamma$ (with $w_{m}^{(S)}=$ $\left.0.00003, s_{m}=0\right)$ for SoRec. The results are shown in Figure 1. As expected, it is important to find the right tradeoff between the social network information and the rating data. While all three models show an improvement in recall compared to No Trust case, it is particularly large for SoRec. SoRec model with our modified training objective function outperforms No Trust by $23.1 \%$ in terms of overall recall and $101.8 \%$ in terms of cold user recall. It shows that social network is very helpful in terms of top-k recommendation especially for recommendation of cold start users. Moreover, recall is even slightly higher for cold users than it is for all users. This may be explained by the fact that cold users have a slight tendency to rate popular items (i.e. items with a large number of ratings), which can naturally be recommended more accurately. In the Epinions data, the average item rated by a cold user has received 102 ratings, while the average item rated by all users has received only 93 ratings. Note that this tendency is much more pronounced in the Flixster data (see Figure 4), where recommendations can be made even more accurately for cold users than for all users (see Figure 3).

The recall test results for the optimal tuning parameters are summarized for all these MF models in Table 1. Table 1 shows that the SoRec model with our modified training objective function achieves the best recall. This may be unexpected, as the SoRec model was found to achieve a worse RMSE than STE in [11], and STE was found to have a worse RMSE than SocialMF in [8]. This result illustrates

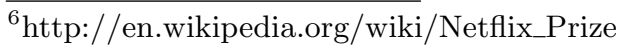

that the best way of combining rating data with social network information concerning the popular RMSE measure is not necessarily the best way to maximize recall.

\begin{tabular}{|l||r|r|r|r|}
\hline \multicolumn{1}{|l||}{$\begin{array}{l}\text { test } \\
\text { users }\end{array}$} & No Trust & SocialMF & STE & SoRec \\
\hline \hline \multicolumn{5}{|c|}{ original training (on observed ratings) } \\
\hline all & $1.9 \%$ & $3.5 \%$ & $2.7 \%$ & $2.6 \%$ \\
\hline cold & $1.5 \%$ & $1.0 \%$ & $2.8 \%$ & $2.9 \%$ \\
\hline \hline \multicolumn{5}{|c|}{ modified training (on all ratings) } \\
\hline all & $26.0 \%$ & $29.1 \%$ & $29.4 \%$ & $32.0 \%$ \\
\hline cold & $16.5 \%$ & $27.9 \%$ & $26.6 \%$ & $33.3 \%$ \\
\hline
\end{tabular}

Table 1: Epinions data: recall (top 500) in percent for three MF models trained with original and modified training objective. 'No Trust' is the baseline MF model that only uses rating data.

\subsubsection{RMSE for MF Models}

Apart from optimizing for recall, we also determined the optimal tuning parameters as to minimize RMSE, and found $\lambda=0.1, r_{m}=4, w_{m}=0, j_{0}=10$, which resulted in the following RMSE values:

- $R M S E=1.174$, if only the rating data is used,

- $R M S E=1.095$, for SocialMF (with $\beta=20$ ),

- $R M S E=1.157$, for STE (with $\alpha=0.5$ ),

- $R M S E=1.117$, for SoRec $\left(\right.$ with $\gamma=50$ and $w_{M}^{(S)}=$ $0)$.

These results are consistent with RMSE results in the in the literature $[8,11,12]$. It verifies that social network information is useful for improving RMSE.

\subsubsection{Recall for NN Models}

As a further comparison, Figures 2 shows the recall test results we obtained for various nearest neighbor models, which are outlined in Section 3.2. To the best of our knowledge, this includes the only top- $k$ approach using social network information [7], the Trust-cf model. In Trust-cf model, $k_{1}$ is set to be 5 which leads to best recall in user based CF. Top500 recommendation result on Epinions dataset of Trust-cf is shown in Figure 2(c).

Among the NN approaches, the one in Figure 2(c) achieves a considerably worse hit ratio than any of the NN approaches 


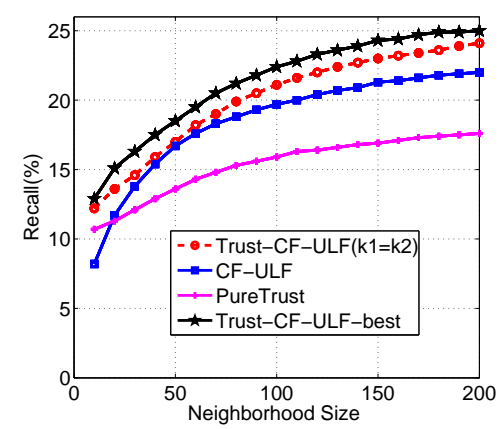

(a) Nearest Neighbor: all users

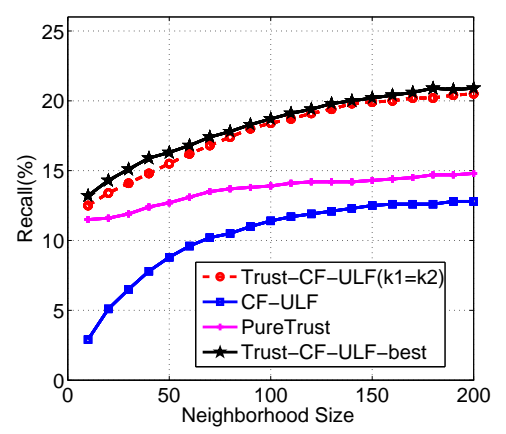

(b) Nearest Neighbor: cold users

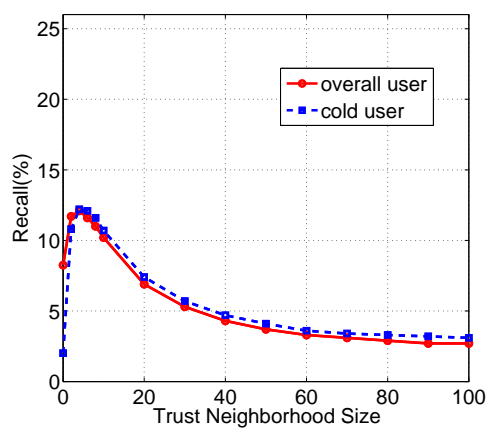

(c) Trust-cf: $k_{1}=5$, varies $k_{2}$

Figure 2: Top-500 Recall by Nearest Neighbor based Models on Epinions dataset

in Figure 2(a) and 2(b). This poor performance of the only published top- $k$ approach (in Figure 2(c)) [7]-is due to the following reason: the NN approach in [7] predicts the rating value of a user in terms of the average rating values of the user's friends-which is obviously based on the observed ratings only. In contrast, the various NN approaches in Figure 2(a) and 2(b) use voting-which is the simplest possible way of accounting for all items, i.e. by counting 0 for an absent rating and counting 1 for an observed relevant rating (with weights defines in Section 3.2). As the rating value is ignored, this is the simplest possible approach to account for all items during training. Though recall of NN based RS is not as good as MF based RS. As we mentioned in Section 3.2, NN based approach enjoys the advantage of incrementally integrating user's new feedbacks while MF based approach is not able to.

\subsection{Experiments on Flixster Dataset}

Despite the different properties of the Epinions and Flixster data sets, the results on the Flixster data confirm our results on the Epinions data. Flixster is a social network site where users add other users to their friend lists to form a social network. Flixster has about one million users, who rate movies and share reviews. The ratings in Flixster have ten discrete values from 0.5 to 5 , with step size of 0.5 . Flixster is different from Epinions in that social relations in Flixster are bi-directional. The Flixster data ${ }^{7}$ used here is from [8]. The Flixster social network has 26.7 million connections. The trace consists of 8.2 million movie ratings on 49,000 movies and 1 million users. The number of users who made at least one rating is 150,000 . Note that a large portion of users in the Flixster data did not rate movies. However, they are useful for social-network-based recommender systems, since a recommendation can be made using the ratings of users who are reachable through the no-rating users. We split the data into a training set and a disjoint test set. For users with less than 10 ratings, we randomly choose one rating and put it into test set. For users with 10 or more than 10 ratings, we randomly chose $5 \%$ as put them into the test set. We further split the test set randomly into two disjoint sets of equal size and report results of testing the second test set akin to Epinions case. We defined rating values of 4 or larger as relevant for computing recall on the test set. We report recall for the top 100 items recommendation. The top- $k$ hit ratio of different $k$ value is presented in Figure 7 .

\footnotetext{
${ }^{7}$ http://www.sfu.ca/ sja25/datasets/
}

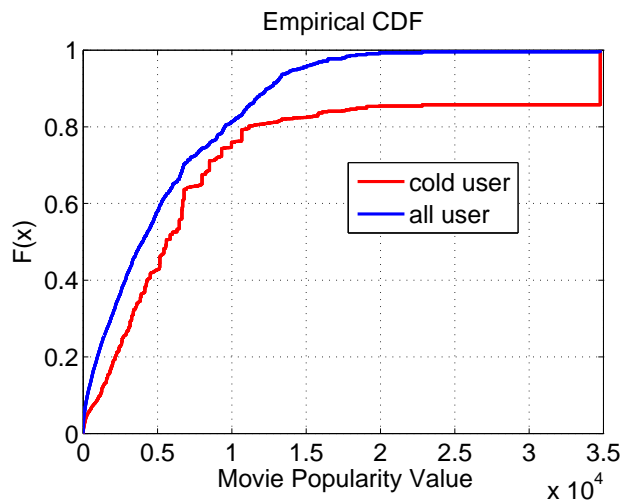

Figure 4: Comparison of cold users' and overall users' tendency to rate popular items in the Flixster data.

Figure 3 shows that recall can be improved by using the social network information in addition to the rating data. The optimal values for the other tuning parameters are: $\lambda=$ $0.1, r_{m}=1$ and $w_{m}=0.2$. The optimal $w_{m}^{(S)}$ and $s_{m}$ are 0.2 and 0 respectively in modified SoRec model. For these optimal training parameters, the recall test results are summarized for all the MF models in Table 2.

\begin{tabular}{|c|c|c|c|c|}
\hline \multirow{2}{*}{$\begin{array}{l}\text { test } \\
\text { users }\end{array}$} & \multicolumn{4}{|c|}{ MF models } \\
\hline & No Trust & SocialMF & STE & SoRec \\
\hline \multicolumn{5}{|c|}{ original training (on observed ratings) } \\
\hline all & $4.4 \%$ & $4.7 \%$ & $5.3 \%$ & $8.2 \%$ \\
\hline cold & $6.3 \%$ & $6.6 \%$ & $7.2 \%$ & $15.4 \%$ \\
\hline \multicolumn{5}{|c|}{ modified training (on all ratings) } \\
\hline all & $44.3 \%$ & $45.2 \%$ & $47.1 \%$ & $49.1 \%$ \\
\hline cold & $30.8 \%$ & $38.3 \%$ & $47.6 \%$ & $59.2 \%$ \\
\hline
\end{tabular}

Table 2: Flixster data: recall (top 100) in percent for three MF models trained with original and modified training objective. 'No Trust' is the baseline MF model that only uses rating data.

As before, SoRec with modified training objective function achieves the largest recall. We can see from Table 2 that SoRec model with our modified training objective function outperforms No Trust by $10.8 \%$ in terms of overall recall and $92.2 \%$ in terms of cold user recall. It again shows that social network is very helpful in terms of top-k recommendation es- 


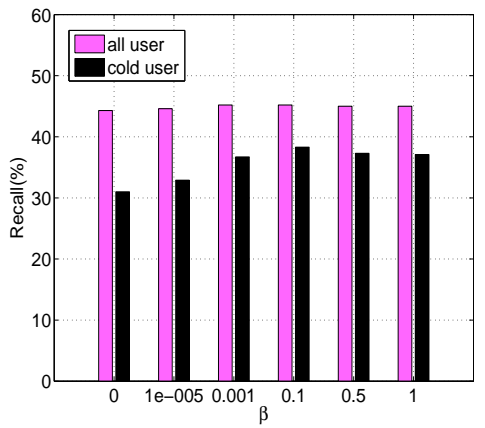

(a) SocialMF model

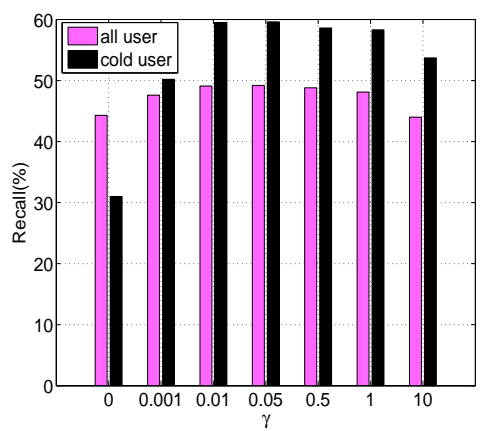

(b) SoRec model

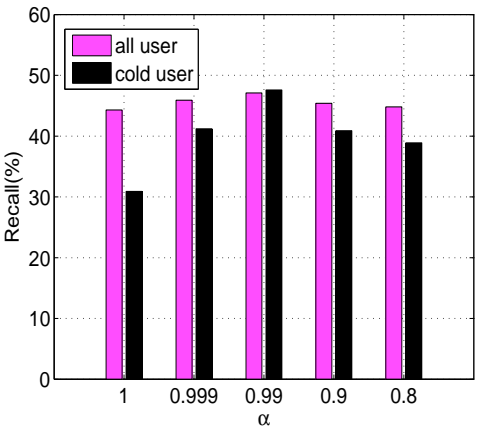

(c) STE model

Figure 3: Top-100 Recall for social-network-based matrix-factorization models on Flixster data.

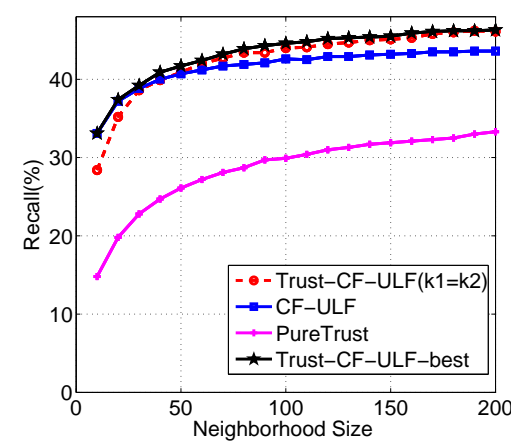

(a) Nearest Neighbor: all users

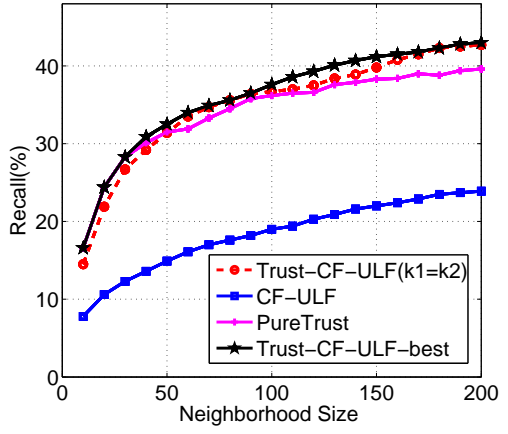

(b) Nearest Neighbor: cold users

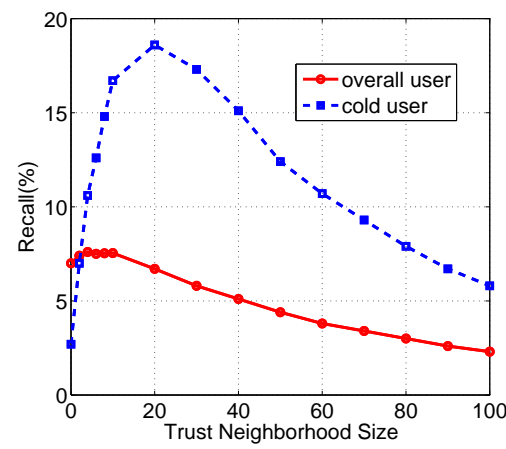

(c) Trust-cf: $k_{1}=20$, varies $k_{2}$

Figure 5: Top-100 Recall by Nearest-Neighbor-based Models on Flixster dataset

pecially for recommendation of cold start users. Note that the improvement for the cold users over all the users is particularly pronounced for the Flixtser data, as cold users have a rather strong tendency to rate popular items, as shown in Figure 4.

In Trust-cf model, $k_{1}$ is set to be 20 which leads to best recall in user based CF. Top-100 recommendation result on Flixster dataset of Trust-cf is shown in Figure 5(c). Akin to Epinions dataset, among the NN approaches, the one in Figure 5(c) achieves a considerably worse hit ratio than any of the NN approaches in Figure 5(a) and 5(b).

\subsection{Impact of Dimensionality and Top- $k$}

Figure 6 and 7 depict recall vs. the top- $k$ number with dimensionality $j_{0}=10$ and $j_{0}=20$ respectively. We can see from Figure 6 and 7 that dimensionality $j_{0}=20$ performs better than $j_{0}=10$. This is because larger dimensionality captures more latent features of users and items and hence improves recall. It should be noted that top- $k$ hit ratio of Flixster data is much more better than Epinions data. Counting that the number of items in Epinions dataset is about two times of Flixster dataset, still, we find that recall of Flixster is more than two times of Epinions for top-5 to top-500 recommendations. This is probably because of the fact that Epinions data is a multi-category data which contains items from many categories(cars, movies, books, software, etc.) while items in Flixster are all movies which makes the recommendation easier in general. Furthermore, users in Flixster dataset averagely have more number of social connections and item ratings compared to Epinions dataset.

\section{CONCLUSIONS}

Social recommendation is prevalent in real-world, but top$\mathrm{k}$ recommendation using online social networks has been insufficiently studied in the recommendation literature. In this paper, we present a comprehensive study on improving the accuracy of top-k recommendation using trust information derived from social networks. We showed that the existing social network based recommender systems can be conveniently tailored for top- $\mathrm{k}$ recommendations by modifying their training objective functions to account for both observed ratings and missing ratings. Through experiments on two large-scale data sets, we made three majors findings: 1)Trust information significantly improves top- $k$ hit ratio when incorporated properly both in MF and NN models; 2)Our proposed social network based NN models and $\mathrm{RS}$ trained with our modified objective function considerably outperform the only published top- $k$ approach using social network information in recent literature [7], an NN approach; 3) among the various ways of combing feedback data with social network information, the one that was found to be worst with respect to RMSE turns out to be the best concerning the top- $k$ hit ratio. This illustrates that the technical details of minimizing RMSE can be very different from the ones that work to optimize the more realistic top- $k$ hit ratio.

Among all NN approaches, Trust-CF-ULF-best performs best in terms of top- $k$ hit ratio. And modified SoRec model is the best among all MF models in terms of hit-ratio. 


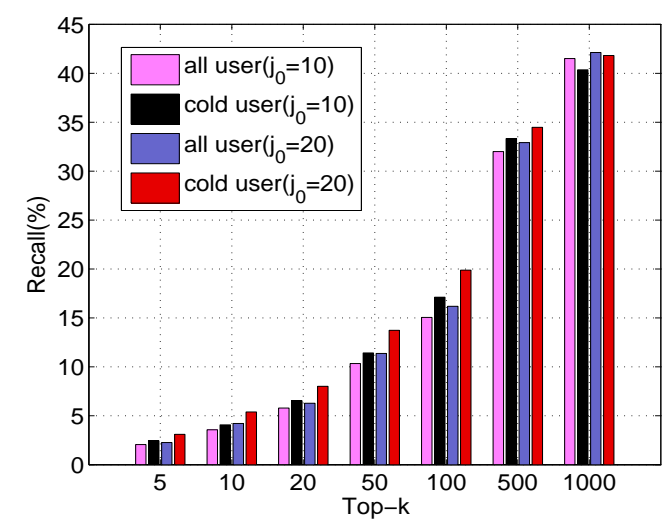

Figure 6: Recall vs Top- $k$ and impact of Dimensionality on Epinions data.

In summary, our work demonstrated that top- $k$ recommendations pose unique challenges, and social trust information, when incorporated properly, can significantly improve the hit ratio of top- $k$ recommendations.

\section{REFERENCES}

[1] G. Adomavicius and A. Tuzhilin. Toward the next generation of recommender systems: A survey of the state-of-the-art and possible extensions. IEEE Transactions on Knowledge and Data Engineering, 17:734-49, 2005.

[2] P. Cremonesi, Y. Koren, and R. Turrin. Performance of recommender algorithms on top-N recommendation tasks. In ACM Conference on Recommender Systems, pages 39-46, 2010.

[3] S. Funk. Netflix update: Try this at home, 2006. http://sifter.org/ simon/journal/20061211.html.

[4] J. A. Golbeck. Computing and applying trust in web-based social networks, 2005.

[5] Y. Hu, Y. Koren, and C. Volinsky. Collaborative filtering for implicit feedback datasets. In IEEE International Conference on Data Mining (ICDM), 2008.

[6] M. Jamali and M. Ester. Trustwalker: a random walk model for combining trust-based and item-based recommendation. In ACM Conference on Knowledge Discovery and Data Mining (KDD), 2009.

[7] M. Jamali and M. Ester. Using a trust network to improve top-n recommendation. In ACM Conference on Recommender Systems (RecSys), 2009.

[8] M. Jamali and M. Ester. A matrix factorization technique with trust propagation for recommendation in social networks. In ACM Conference on Recommender Systems (RecSys), 2010.

[9] R. Keshavan, A. Montanari, and S. Oh. Matrix completion from noisy entries. Journal of Machine Learning Research, 11:2057-78, 2010.

[10] Y. Koren. Factorization meets the neighborhood: a multifaceted collaborative filtering model. In $A C M$ Conference on Knowledge Discovery and Data Mining, pages 426-34, 2008.

[11] H. Ma, I. King, and M. R. Lyu. Learning to recommend with social trust ensemble. In $A C M$

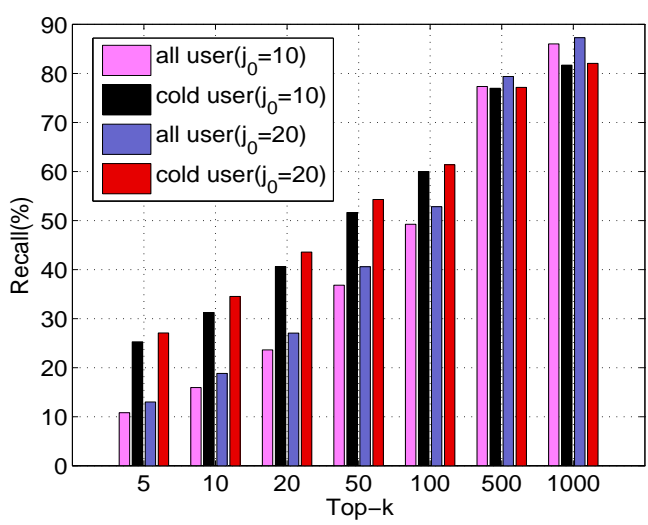

Figure 7: Recall vs Top- $k$ and impact of Dimensionality on Flixster data.

conference on Research and development in information retrieval (SIGIR), 2009.

[12] H. Ma, H. Yang, M. R. Lyu, and I. King. Sorec: Social recommendation using probabilistic matrix factorization. In International Conference on Information and Knowledge Management (CIKM), 2008.

[13] B. Marlin and R. Zemel. Collaborative prediction and ranking with non-random missing data. In $A C M$ Conference on Recommender Systems (RecSys), 2009.

[14] B. Marlin, R. Zemel, S. Roweis, and M. Slaney. Collaborative filtering and the missing at random assumption. In Conference on Uncertainty in Artificial Intelligence (UAI), 2007.

[15] P. Massa and P. Avesani. Trust-aware recommender systems. In ACM Conference on Recommender Systems (RecSys), 2007.

[16] R. Pan, Y. Zhou, B. Cao, N. Liu, R. Lukose, M. Scholz, and Q. Yang. One-class collaborative filtering. In IEEE International Conference on Data Mining (ICDM), 2008.

[17] A. Paterek. Improving regularized singular value decomposition for collaborative filtering. In $K D D C u p$, 2007.

[18] R. Salakhutdinov, A. Mnih, and G. Hinton. Restricted Boltzmann machines for collaborative filtering. In International Conference on Machine Learning (ICML), 2007.

[19] R. Salakhutdinov and N. Srebro. Collaborative filtering in a non-uniform world: Learning with the weighted trace norm. In Advances in Neural Information Processing Systems 24 (NIPS), 2010.

[20] N. Srebro and T. Jaakkola. Weighted low-rank approximations. In International Conference on Machine Learning (ICML), pages 720-7, 2003.

[21] H. Steck. Training and testing of recommender systems on data missing not at random. In $A C M$ Conference on Knowledge Discovery and Data Mining, pages 713-22, 2010.

[22] M. Richardson and P. Domingos. Mining Knowledge-Sharing Sites for Viral Marketing, In ACM Conference on Knowledge Discovery and Data mining, 2002. 